\title{
A Common Coupled Fixed Point Theorem in Complex Valued Metric Space
}

\author{
Savitri \\ Department of Mathematics \\ DCR University of Science and Technology \\ Murthal, Sonepat, Haryana (India)
}

\author{
Nawneet Hooda \\ Department of Mathematics \\ DCR University of Science and Technology \\ Murthal, Sonepat, Haryana (India)
}

\begin{abstract}
The aim of this paper is to establish a unique common coupled fixed point theorem for two mappings satisfying a rational inequality in complex valued metric space.
\end{abstract}

\section{General Terms}

47H10, 54H 25

\section{Keywords}

Coupled fixed point, complex valued metric space, Cauchy sequence, Convergent sequence, complete complex valued metric space.

\section{INTRODUCTION AND PRELIMINARIES}

Azam, Fisher and Khan [6] introduced the concept of complex-valued metric spaces and established the existence of common fixed point theorems for a pair of contractive type mappings involving rational expressions. These results were further extended and generalized by Rouzkard and Imdad [10]. Klin-Eam and Suanoom [9] extended the concept of complex valued metric spaces and generalized the results of Azam et al. [1] and Rouzkard and Imdad [10]. Subsequently, several authors studied the existence and uniqueness of fixed points in complex valued spaces $[1-5,7,8,11,12]$.

Consistent with Azam et al. [6], the following definitions and notations will be needed in the sequel.

Let $\square$ be the set of complex numbers and $z_{1}, z_{2} \in \square$. Define a partial order $\uparrow$ on $\square$ as follows:

$z_{1}$ đ $z_{2}$ if and only if $\operatorname{Re}\left(z_{1}\right) \leq \operatorname{Re}\left(z_{2}\right)$ and $\operatorname{Im}\left(z_{1}\right) \leq \operatorname{Im}\left(z_{2}\right)$

If follows that $z_{1} \uparrow z_{2}$ if and only if one of the following conditions is satisfied:

(i) $\operatorname{Re}\left(z_{1}\right)=\operatorname{Re}\left(z_{2}\right)$ and $\operatorname{Im}\left(z_{1}\right)<\operatorname{Im}\left(z_{2}\right)$,

(ii) $\operatorname{Re}\left(z_{1}\right)<\operatorname{Re}\left(z_{2}\right)$ and $\operatorname{Im}\left(z_{1}\right)=\operatorname{Im}\left(z_{2}\right)$,

(iii) $\operatorname{Re}\left(z_{1}\right)<\operatorname{Re}\left(z_{2}\right)$ and $\operatorname{Im}\left(z_{1}\right)<\operatorname{Im}\left(z_{2}\right)$,

(iv) $\operatorname{Re}\left(z_{1}\right)=\operatorname{Re}\left(z_{2}\right)$ and $\operatorname{Im}\left(z_{1}\right)=\operatorname{Im}\left(z_{2}\right)$,

In particular, we will write $z_{1} Æ z_{2}$ if $z_{1} \neq z_{2}$ and one of (i), (ii), and (iii) is satisfied and we will write $z_{1} \mathrm{p} z_{2}$ if only (iii) satisfied.

Definition 1.1. Let $X$ be a nonempty set. Suppose that the mapping $d: X \times X \rightarrow \square$ satisfies: (i) 0 I $d(x, y)$ for all $x, y \in X$; and $d(x, y)=0$ if and only if $x=y$,

(ii) $d(x, y)=d(y, x)$ for all $x, y \in X$,

(iii) $\quad d(x, y) \llbracket d(x, z)+d(z, y)$ for all $x, y, z \in X$

Then $d$ is called a complex valued metric on $X$ and $(X, d)$ is called a complex valued metric space.

Lemma 1.1. Let $(X, d)$ be a complex-valued metric space and let $\left\{x_{n}\right\}$ be a sequence in $X$. Then $\left\{x_{n}\right\}$ converges to $X$ iff $\left|d\left(x_{n}, x\right)\right| \rightarrow 0$ as $n \rightarrow \infty$.

Lemma 1.2. Let $(X, d)$ be a complex-valued metric space and let $\left\{x_{n}\right\}$ is a Cauchy sequence iff $\left|d\left(x_{n}, x_{n+m}\right)\right| \rightarrow 0$ as $n \rightarrow \infty$.

Definition 1.2. An element $(x, y) \in X \times X$ is called a coupled fixed point of the mapping $T: X \times X \rightarrow X$ if

$$
T(x, y)=x \text { and } T(y, x)=y .
$$

Definition 1.3. Let $(X, d)$ be a complex-valued metric space. If every Cauchy sequence is convergent in $(X, d)$, then $(X, d)$ is called a complete complex-valued metric space.

\section{MAIN RESULTS}

Theorem 2.1. Let $(X, d)$ be a complete complex-valued metric space and $S, T: X^{2} \rightarrow X$ are mapping satisfying

$$
\begin{array}{r}
{\left[d\left(x_{1}, S\left(x_{1}, x_{2}\right)\right) d\left(x_{1}, T\left(y_{1}, y_{2}\right)\right)\right.} \\
d\left(S\left(x_{1}, x_{2}\right), T\left(y_{1}, y_{2}\right)\right) \leq \alpha \frac{\left.+d\left(x_{1}, T\left(y_{1}, y_{2}\right)\right) d\left(y_{1}, S\left(x_{1}, x_{2}\right)\right)\right]}{d\left(x_{1}, T\left(y_{1}, y_{2}\right)\right)+d\left(y_{1}, S\left(x_{1}, x_{2}\right)\right)}
\end{array}
$$

for all $x_{1}, x_{2}, y_{1}, y_{2} \in X$, where $0 \leq \alpha<1$.

Then $S$ and $T$ have a unique common coupled fixed point.

Proof. For any arbitrary points $x_{0}, y_{0} \in X$, construct the sequence $\left\{x_{n}\right\},\left\{y_{n}\right\}$ in $X$ such that

$x_{n+1}=S\left(x_{n}, y_{n}\right), x_{n+2}=T\left(x_{n+1}, y_{n+1}\right)$ and $y_{n+1}=S\left(y_{n}, x_{n}\right)$, $y_{n+2}=T\left(y_{n+1}, x_{n+1}\right), \quad$ for all $n=0,1,2, \ldots$

Now 


$$
\begin{aligned}
& d\left(x_{n+1}, x_{n+2}\right) \\
& =d\left(S\left(x_{n}, y_{n}\right), T\left(x_{n+1}, y_{n+1}\right)\right) \\
& \leq \alpha \frac{\left(\begin{array}{l}
{\left[\left(x_{n}, S\left(x_{n}, y_{n}\right)\right) d\left(x_{n}, T\left(x_{n+1}, y_{n+1}\right)\right)\right.} \\
\left.+d\left(x_{n+1}, T\left(x_{n+1}, y_{n+1}\right)\right) d\left(x_{n+1}, S\left(x_{n}, y_{n}\right)\right)\right]
\end{array}\right)}{d\left(x_{n}, T\left(x_{n+1}, y_{n+1}\right)\right)+d\left(x_{n+1}, S\left(x_{n}, y_{n}\right)\right)} \\
& =\alpha \frac{\left[d\left(x_{n}, x_{n+1}\right) d\left(x_{n}, x_{n+2}\right)+d\left(x_{n+1}, x_{n+2}\right) d\left(x_{n+1}, x_{n+1}\right)\right]}{d\left(x_{n}, x_{n+2}\right)+d\left(x_{n+1}, x_{n+1}\right)} \\
& =\alpha d\left(x_{n}, x_{n+1}\right) \quad \text { for all } n \geq 0
\end{aligned}
$$

Hence $d\left(x_{n+1}, x_{n+2}\right) \leq \alpha d\left(x_{n}, x_{n+1}\right) \leq \ldots \leq \alpha^{n+1} d\left(x_{0}, x_{1}\right)$

Now for $m>n$, we have

$$
\begin{aligned}
d\left(x_{m}, x_{n}\right) & \leq d\left(x_{n}, x_{n+1}\right)+d\left(x_{n+1}, x_{n+2}\right)+\ldots+d\left(x_{m-1}, x_{n}\right) \\
& \leq \alpha^{n} d\left(x_{0}, x_{1}\right)+\alpha^{n+1} d\left(x_{0}, x_{1}\right)+\ldots+\alpha^{m-1} d\left(x_{0}, x_{1}\right) \\
& \leq \frac{\alpha^{n}}{1-\alpha} d\left(x_{0}, x_{1}\right)
\end{aligned}
$$

implies

$$
\left|d\left(x_{n}, x_{n}\right)\right| \leq \frac{\alpha^{n}}{1-\alpha}\left|d\left(x_{0}, x_{1}\right)\right|
$$

which implies that $\left|d\left(x_{n}, x_{m}\right)\right| \rightarrow 0$ as $n, m \rightarrow \infty$.

Hence $\left\{x_{n}\right\}$ is Cauchy sequence.

Also

$$
\begin{aligned}
& d\left(y_{n+1}, y_{n+2}\right) \\
& =d\left(S\left(y_{n}, x_{n}\right), T\left(y_{n+1}, x_{n+1}\right)\right) \\
& \leq \alpha \frac{\left(\begin{array}{l}
{\left[d\left(y_{n}, S\left(y_{n}, x_{n}\right)\right) d\left(y_{n+1}, T\left(y_{n+1}, x_{n+1}\right)\right)\right.} \\
\left.+d\left(y_{n+1}, T\left(y_{n+1}, x_{n+1}\right)\right) d\left(y_{n+1}, S\left(y_{n}, x_{n}\right)\right)\right]
\end{array}\right)}{d\left(y_{n}, T\left(y_{n+1}, x_{n+1}\right)\right)+d\left(y_{n+1}, S\left(y_{n}, x_{n}\right)\right)} \\
& =\alpha \frac{\left[d\left(y_{n}, y_{n+1}\right) d\left(y_{n+1}, y_{n+2}\right)+d\left(y_{n+1}, y_{n+2}\right) d\left(y_{n+1}, y_{n+1}\right)\right]}{d\left(y_{n}, y_{n+2}\right)+d\left(y_{n+1}, y_{n+1}\right)} \\
& \leq \alpha d\left(y_{n}, y_{n+1}\right)
\end{aligned}
$$

implies

$$
d\left(y_{n+1}, y_{n+2}\right) \leq \alpha d\left(y_{n}, y_{n+1}\right) \quad \text { for all } n \geq 0
$$

Hence

$$
\begin{aligned}
d\left(y_{n+1}, y_{n+2}\right) & \leq \alpha d\left(y_{n}, y_{n+1}\right) \\
& \leq \alpha^{2}\left(y_{n-1}, y_{n}\right) \leq \ldots \leq \alpha^{n+1} d\left(y_{0}, y_{1}\right)
\end{aligned}
$$

For $m>n$, we have

$$
\begin{aligned}
d\left(y_{m}, y_{n}\right) & \leq d\left(y_{n}, y_{n+1}\right)+d\left(y_{n+1}, y_{n+2}\right)+\ldots+d\left(y_{m-1}, y_{m}\right) \\
& \leq \alpha^{n} d\left(y_{0}, y_{1}\right)+\alpha^{n+1} d\left(y_{0}, y_{1}\right)+\ldots+\alpha^{m-1} d\left(y_{0}, y_{1}\right) \\
& \leq \frac{\alpha^{n}}{1-\alpha} d\left(y_{0}, y_{1}\right)
\end{aligned}
$$

implies

$$
\left|d\left(y_{m}, y_{n}\right)\right| \leq \frac{\alpha^{n}}{1-\alpha}\left|d\left(y_{0}, y_{1}\right)\right|
$$

implies

$$
\left|d\left(y_{m}, y_{n}\right)\right| \rightarrow 0 \text { as } n, m \rightarrow \infty
$$

implies

$$
\left\{y_{n}\right\} \text { is also a Cauchy sequence. }
$$

So, $\left\{x_{n}\right\},\left\{y_{n}\right\}$ are Cauchy sequences in $X$. But $X$ is complete so $\exists x, y \in X$ such that $\left\{x_{n}\right\},\left\{y_{n}\right\}$ converges to $x, y$ respectively.

Now we will show that $S(x, y)=x$. If not then $\exists z \in c$ such that

$$
d(S(x, y), x)=z \text { where }|z|>0 .
$$

Now

$$
\begin{aligned}
z & \leq d\left(x, x_{n+2}\right)+d\left(x_{n+2}, S(x, y)\right) \\
& =d\left(x, x_{n+2}\right)+d\left(S(x, y), T\left(x_{n+1}, y_{n+1}\right)\right) \\
& \leq d\left(x, x_{n+2}\right)+\alpha \frac{\left(\begin{array}{l}
{\left[d(x, S(x, y)) d\left(x, T\left(x_{n+1}, y_{n+1}\right)\right)\right.} \\
\left.+d\left(x_{n+1}, T\left(x_{n+1}, y_{n+1}\right)\right) d\left(x_{n+1}, S(x, y)\right)\right]
\end{array}\right)}{d\left(x, T\left(x_{n+1}, y_{n+1}\right)\right)+d\left(x_{n+1}, S(x, y)\right)}
\end{aligned}
$$

implies

$$
|z| \leq\left|d\left(x, x_{n+2}\right)\right|+\alpha \frac{\left(\begin{array}{l}
{\left[|z|\left|d\left(x, x_{n+2}\right)\right|\right.} \\
\left.\left.+\mid d\left(x_{n+1}, x_{n+2}\right)\right)|| d\left(x_{n+1}, S(x, y)\right) \mid\right]
\end{array}\right)}{\left|d\left(x, x_{n+2}\right)\right|+\left|d\left(x_{n+1}, S(x, y)\right)\right|} .
$$

On taking limit as $n \rightarrow \infty$ we get $|z| \leq 0$, a contradiction.

So $|z|=0$. Hence $S(x, y)=x$.

Also if possible, let $d(S(y, x), y)=t$ where $|t|>0$. Now

$$
\begin{aligned}
t & \leq d\left(y, y_{n+2}\right)+d\left(y_{n+2}, S(y, x)\right) \\
& =d\left(y, y_{n+2}\right)+d\left(S(y, x), T\left(y_{n+1}, x_{n+1}\right)\right) \\
& \leq d\left(y, y_{n+2}\right)+\alpha \frac{\left(\begin{array}{l}
{\left[d(y, S(y, x)) d\left(y, T\left(y_{n+1}, x_{n+1}\right)\right)\right.} \\
\left.+d\left(y_{n+1}, T\left(y_{n+1}, x_{n+1}\right)\right) d\left(y_{n+1}, S(y, x)\right)\right]
\end{array}\right)}{d\left(y, T\left(y_{n+1}, x_{n+1}\right)\right)+d\left(y_{n+1}, S(y, x)\right)} \\
& =d\left(y, y_{n+2}\right)+\alpha \frac{\left[t d\left(y, y_{n+2}\right)+d\left(y_{n+1}, y_{n+2}\right) d\left(y_{n+1}, S(y, x)\right)\right]}{d\left(y, y_{n+2}\right)+d\left(y_{n+1}, S(y, x)\right)}
\end{aligned}
$$

implies

$$
|t| \leq\left|d\left(y, y_{n+2}\right)\right|+\alpha \frac{\left(\begin{array}{l}
{\left[|t|\left|d\left(y, y_{n+2}\right)\right|\right.} \\
\left.\left.+\mid d\left(y_{n+1}, y_{n+2}\right)\right)|| d\left(y_{n+1}, S(y, x)\right) \mid\right]
\end{array}\right)}{\left|d\left(y, y_{n+2}\right)\right|+\left|d\left(y_{n+1}, S(y, x)\right)\right|} .
$$

On taking limit as $n \rightarrow \infty$ we get $|t| \leq 0$, a contradiction.

So $t=0$. Hence $S(y, x)=y$.

Similarly $T(x, y)=x$ and $T(y, x)=y$.

Hence $(x, y)$ is the common coupled fixed point of $S$ and $T$

For uniqueness. Let $(u, v)$ is another common fixed point of $S$ and $T$. Then 


$$
\begin{aligned}
d(x, u) & =(S(x, y), T(u, v)) \\
& \leq \alpha \frac{\left(\begin{array}{l}
{[d(x, S(x, y)) d(x, T(u, v))} \\
+d(u, T(u, v)) d(u, S(x, y))]
\end{array}\right)}{d(x, T(u, v))+d(u, S(x, y))}
\end{aligned}
$$

[4] Arshad M, Karapinar E, Ahmad J (2013), Some unique fixed point theorems for rational contractions in partially ordered metric spaces, J. Inequl. Appl. Article ID 248.

[5] Azam A, Ahmad J, Kuman P (2013), Common fixed point theorems for multi-valued mappings in complexvalued metric spaces, J. Inequal. Appl., Article ID 578.

implies

$$
d(x, u) \leq 0
$$

implies

$$
x=u
$$

Similarly $y=v$.

So, $(x, y)$ is the unique common coupled fixed point for $S$ and $T$.

\section{REFERENCES}

[1] Abbas M, Arsad M, Azam A (2013), Fixed points of asymptotically regular mappings in complex-valued metric spaces, Georgian Math. J., 20 (2), 213-221.

[2] Abbas M. Fisher B, Nazir T (2012), Well-posedness and periodic point property of mappings satisfying a rational inequality in an ordered complex valued metric space, Sci. Stud. Res., Ser. Math. Inform., 22 (1), 5-24.

[3] Ahmad J, Klin-Eam C, Azam A (2013), Common fixed points for multivalued mappings in complex valued metric spaces with applications, Abstr Appl. Anal. Article ID 854965.
[6] Azam A, Fisher B, Khan M (2011), Common fixed poin theorems in complex valued metric spaces, Numerical Functional Analysis and Optimization, 32 (3), 243-253.

[7] Fisher B (1981), Four mappings with a common fixed point, J. Univ. Kuwait Sci., 8, 131-139.

[8] Jungck G, Rohades BE (1988), Fixed point for set valued functions without continuity, Indian J. Pure Appl. Math., 29 (3), 227-238.

[9] Klin-Eam C, Suanoom C (2013), Some common fixedpoint theorems for generalized-contractive-type mappings on complex-valued metric space, Abstr. Appl. Anal. Article ID 604215.

[10] Rouzkard F, Imdad M (2012), Some common fixed point theorems on complex valued metric spaces, Comput. Math. Appl., 64 (6), 1866-1874.

[11] Bhatt S, Chaukiyal S, Dimri RC (2011), Common fixed point of mappings satisfying rational inequality in complex valued metric space, International Journal of Pure and Applied Mathematics, 73 (2), 159-164.

[12] Sintunavart W, Kuman P (2012), Generalized Common fixed point theorems in complex valued metric spaces and application, Journal of Inequalities and Applications, Article ID 84. 